\title{
FRACTIONAL LAPLACE MOTION
}

\author{
T. J. KOZUBOWSKI, ${ }^{*}$ University of Nevada at Reno \\ M. M. MEERSCHAERT, ${ }^{* *}$ University of Otago \\ K. PODGÓRSKI, ${ }^{* * *}$ Indiana University-Purdue University Indianapolis
}

\begin{abstract}
Fractional Laplace motion is obtained by subordinating fractional Brownian motion to a gamma process. Used recently to model hydraulic conductivity fields in geophysics, it might also prove useful in modeling financial time series. Its one-dimensional distributions are scale mixtures of normal laws, where the stochastic variance has the generalized gamma distribution. These one-dimensional distributions are more peaked at the mode than is a Gaussian distribution, and their tails are heavier. In this paper we derive the basic properties of the process, including a new property called stochastic selfsimilarity. We also study the corresponding fractional Laplace noise, which may exhibit long-range dependence. Finally, we discuss practical methods for simulation.
\end{abstract}

Keywords: Compound process; fractional Brownian motion; G-type distribution; gamma process; generalized gamma distribution; infinite divisibility; long-range dependence; scaling; self-affinity; self-similarity; subordination

2000 Mathematics Subject Classification: Primary 60G07; 60G18; 60E07

\section{Introduction}

Recent research in geophysics has proposed a model in which hydraulic conductivity is obtained by subordinating fractional Brownian motion (FBM) to a gamma process. This process was termed fractional Laplace motion (FLM) since (in the uncorrelated case) at some scale the increments of the process have a Laplace (two-sided exponential) distribution. Logarithms of hydraulic conductivity data exhibit a correlation structure similar to that of fractional Brownian motion, but the increment distributions are more peaked at the mode, with heavier tails. Furthermore, the increment distributions tend to a Gaussian distribution as the spacing of the increments widens. The FLM process reproduces all of these features. Similar features are also present in some financial time series, suggesting that FLM may find wider applications.

Fractional Brownian motion with parameter $H \in(0,1)$ is a centered Gaussian process $\left\{B_{H}(t), t \geq 0\right\}$ with $B_{H}(0)=0$ and covariance function

$$
\mathrm{E}\left(B_{H}(t) B_{H}(s)\right)=\frac{\sigma^{2}}{2}\left(|t|^{2 H}+|s|^{2 H}-|t-s|^{2 H}\right), \quad t, s \geq 0,
$$

Received 16 December 2004; revision received 22 December 2005.

* Postal address: Department of Mathematics and Statistics, Mail Stop 84, University of Nevada, Reno, NV 89557, USA. Email address: tkozubow@unr.edu

Partially supported by NSF grant DMS-0139927.

** Postal address: Department of Mathematics and Statistics, University of Otago, PO Box 56, Dunedin 9001, New Zealand.

Partially supported by NSF grants DMS-0139927 and DMS-0417869 and the Marsden Fund administered by the Royal Society of New Zealand.

*** Postal address: Department of Mathematical Sciences, IUPUI, Indianapolis, IN 46202, USA. 
where $\sigma^{2}=\operatorname{var}\left(B_{H}(1)\right)$. FBM exhibits self-similarity with parameter $H$; i.e. for each $c>0$ we have

$$
\left\{B_{H}(c t), t \geq 0\right\} \stackrel{\mathrm{D}}{=}\left\{c^{H} B_{H}(t), t \geq 0\right\},
$$

in the sense that all finite-dimensional distributions of the two processes are the same (here $\stackrel{\text { D }}{=}$ denotes equality in distribution). Moreover, the stationary increment process $\left\{Z_{k}=B_{H}(k)-\right.$ $\left.B_{H}(k-1)\right\}$, called fractional Gaussian noise, exhibits long-range dependence when $H \in\left(\frac{1}{2}, 1\right)$, i.e. its covariance function $\gamma(k)=\mathrm{E}\left(Z_{i} Z_{i+k}\right)$ tends to 0 so slowly that the series $\sum_{k=1}^{\infty} \gamma(k)$ diverges [6, pp. 41-66], [17], [36].

Historical return distributions (on a short time scale) of currency exchange rates, interest rates, commodities, equity markets, and other financial data often have 'fatter tails' and are more peaked around the mode than is predicted by the normal law [15], [21]. Similar observations have been made in geophysics [27], [29], [30]. The Laplace distribution with probability density function $f(x)=\mathrm{e}^{-|x| / \sigma} /(2 \sigma)$ provides one alternative model that captures the higher peaks and wider tails [20, p. 16]. Given a geometric random variable $N_{p}$ with the probability density function

$$
\mathrm{P}\left(N_{p}=k\right)=p(1-p)^{k-1}, \quad k \in \mathbb{N},
$$

the (geometric) sum of independent, identically distributed Laplace variables (independent of $N_{p}$ ) is Laplace:

$$
\sqrt{p} \sum_{j=1}^{N_{p}} X_{j} \stackrel{\mathrm{D}}{=} X_{1}, \quad p \in(0,1) .
$$

The corresponding limit theorem says that the geometric sum in (1.3) with independent, identically distributed symmetric $X_{j}$ with finite second moments converges in distribution to the Laplace variable as $p \rightarrow 0$ [20, p. 30]. Moreover, the Laplace distribution is infinitely divisible and a corresponding Lévy motion can also be represented as a Brownian motion $\left\{B_{H}(t), t \geq 0\right\}\left(H=\frac{1}{2}\right)$ subordinated to a gamma process $\{\Gamma(t), t \geq 0\}$ :

$$
\{X(t), t \geq 0\} \stackrel{\mathrm{D}}{=}\left\{B_{H}\left(\Gamma_{t}\right), t \geq 0\right\} .
$$

The Lévy process $\left\{\Gamma_{t}, t \geq 0\right\}$ starts at the origin, and the distribution of the increment $\Gamma_{t+s}-\Gamma_{t}$ has a gamma distribution, $\mathscr{g}(\alpha, \beta)$, with shape parameter $\alpha=s$ and scale $\beta=1$, whose probability density function is

$$
f(x)=\frac{1}{\beta^{\alpha} \Gamma(\alpha)} x^{\alpha-1} \mathrm{e}^{-x / \beta}, \quad x>0 .
$$

Consequently, the marginal distributions of the Laplace motion are scale mixtures of normal distributions of the form

$$
X(t) \stackrel{\mathrm{D}}{=} \sigma G_{t}^{H} Z=\left(\sigma^{2} G_{t}^{2 H}\right)^{1 / 2} Z,
$$

where $H=\frac{1}{2}, Z$ is a standard normal variable, and $G_{t}$ has the gamma distribution $g(t, 1)$. In the finance literature this is known as stochastic volatility, since (informally) the variance of the normal variable is random [21].

The aim of this paper is to extend the Laplace motion model to allow dependence between the increments. We define this process by subordinating fractional Brownian motion with $H \in(0,1)$ to the gamma process via (1.4), and call it fractional Laplace motion. The marginal distributions of this process are still of the form (1.5), but now the stochastic variance $G_{t}^{2 H}$ has the generalized gamma distribution $[18, \mathrm{p} .388]$. The subordinator $\Gamma_{t}$ corresponds to a 
(random) time transformation. In financial applications, the subordinator may correspond to the trading time or volume (see, e.g. [15] and [16]). In geophysics, it may represent the number of depositional features over a distance $t$ [27].

Our paper is organized as follows. In Section 2 we formally define the FLM and study its basic properties. These include the covariance structure as well as densities, moments, stochastic representations, and tail behavior of one-dimensional laws. In Section 3 we study the corresponding fractional Laplace noise and discuss long-range dependence. Section 4 is devoted to the discussion of further properties of infinite divisibility and stochastic selfsimilarity. There we show that FLM is stochastically self-similar with respect to a family of negative binomial subordinators. In Section 5 we discuss methods for simulating FLM and present sample paths for representative values of parameters. Proofs and auxiliary results are collected in Section 6.

\section{Fractional Laplace motion and its basic properties}

Let $\left\{B_{H}(t), t \geq 0\right\}$ be an FBM with the covariance function (1.1), denoted by $\mathcal{F} \mathcal{B} \mathcal{M}_{H}(\sigma)$. Let $\left\{\Gamma_{t}, t \geq 0\right\}$ be a gamma process with parameter $v>0$, i.e. a Lévy process such that the increment $\Gamma_{t+s}-\Gamma_{t}$ has distribution $g(s / v, 1)$ (a standard gamma process as discussed in the introduction has $v=1)$. We now define a new process, $\{X(t), t \geq 0\}$, by subordinating $B_{H}$ to $\Gamma_{t}$, as in (1.4). This process is the FLM, which we denote by $\mathcal{F} \mathcal{L} \mathcal{M}_{H}(\sigma, v)$. Note that if $X \stackrel{\mathrm{D}}{=} \mathcal{F} \mathcal{L} \mathcal{M}(\sigma, 1)$ then $Y=X(\cdot / v) \stackrel{\mathrm{D}}{=} \mathcal{F} \mathcal{L} \mathcal{M}(\sigma, v)$, meaning that $v$ is a time scale parameter. A standard fractional Laplace motion has $\sigma=v=1$, and is denoted by $\mathcal{F} \mathscr{L} \mathcal{M}_{H}$.

\subsection{One-dimensional distributions}

In this subsection we set $\sigma=1$. It is easy to see that the one-dimensional distributions of FLM given by (1.4) are scale mixtures of normal distributions of the form (1.5), where $Z$ and $G_{t}$ are independent, $Z$ is standard normal, and $G_{t}$ has the gamma distribution $g(t / v, 1)$. In the notation of Johnson et al. [18], the 'stochastic variance' $G_{t}^{2 H}$ has the generalized gamma distribution introduced by Amoroso [1] and given by the probability density function

$$
f(x)=\frac{\gamma x^{\alpha \gamma-1}}{c^{\alpha \gamma} \Gamma(\alpha)} \mathrm{e}^{-(x / c)^{\gamma}}, \quad x>0,
$$

with scale $c=1$ and shape parameters $\alpha=t / v$ and $\gamma=0.5 / H$.

Remark 2.1. When $H=\frac{1}{2}$ and the stochastic variance has a gamma distribution, the distribution of $X(t)$ has been referred to as the variance-gamma distribution [23] or the normalgamma distribution [7], in analogy to the normal-inverse Gaussian distribution, which arises when the stochastic variance has the inverse Gaussian distribution. This model leads to the improvements in option pricing theory reported by Madan et al. [22]. In the general case the term is variance-generalized gamma distribution [21]. Such normal mixtures are also known as G-type distributions when the stochastic variance is infinitely divisible [24], [25], [26, p. 39], [33].

Representation (1.5), along with well-known expressions for absolute moments of gamma and normal distributions, leads to the following result.

Proposition 2.1. If $\{X(t), t \geq 0\} \stackrel{\mathrm{D}}{=} \mathcal{F} \mathcal{L}_{\mathcal{M}}(1, v)$ then

$$
\mathrm{E}\left(|X(t)|^{q}\right)=c_{q} \frac{\Gamma(H q+t / \nu)}{\Gamma(t / \nu)}, \quad \text { with } c_{q}=\sqrt{\frac{2^{q}}{\pi}} \Gamma\left(\frac{1+q}{2}\right) .
$$


Remark 2.2. Note that the quantity in (2.2) is linear in $t$ when $q=1 / H$ (in particular, the variance of $X(t)$ is linear in $t$ when $H=\frac{1}{2}$ ). Moreover, this is different than the power law scaling of the FBM, where $\mathrm{E}\left(\left|B_{H}(t)\right|^{q}\right)=c_{q} t^{q H}$. However, the asymptotic properties of the gamma function do yield $\mathrm{E}\left(|X(t)|^{q}\right) \sim c_{q}(t / v)^{q H}$ as $t \rightarrow \infty$, which reflects the fact that the increments of the FLM become Gaussian with an increasing lag (as $t \rightarrow \infty$ we have $G_{t} / t \stackrel{\mathrm{D}}{\rightarrow} 1 / \nu$, whence, by $(1.5), X(t) / t \stackrel{\mathrm{D}}{\rightarrow}(1 / v)^{H} Z, \stackrel{\mathrm{D}}{\rightarrow}$ ' denoting convergence in distribution).

Remark 2.3. Using Proposition 2.1, we find the excess kurtosis of $X(t)$ to be

$$
\gamma_{2}=\frac{\mathrm{E}\left([X(t)-\mathrm{E} X(t)]^{4}\right)}{\mathrm{E}\left([X(t)-\mathrm{E} X(t)]^{2}\right)^{2}}-3=3\left(\frac{\Gamma(4 H+t / v) \Gamma(t / v)}{\Gamma(2 H+t / v)^{2}}-1\right) .
$$

It appears that the ratio of the gamma functions above is monotonically decreasing from $\infty$ to 0 as $t$ varies from 0 to $\infty$. The variables $X(t)$ are thus leptokurtic $\left(\gamma_{2}>0\right)$, indicating heavier tails and a larger degree of peakedness as compared to the normal distribution, and become less leptokurtic with increasing $t$ (eventually approaching the Gaussian case as $t \rightarrow \infty$ ) and more leptokurtic with decreasing $t$.

The form of the one-dimensional marginal density of $X(t)$ follows easily from conditioning on $G_{t}$ in (1.5) as shown in the next result.

Proposition 2.2. The density function of $X(t)$ defined by $(1.5)$ is

$$
f_{t}(x)=\frac{1}{\sqrt{2 \pi} \Gamma(t / v)} \int_{0}^{\infty} y^{t / \nu-H-1} \mathrm{e}^{-x^{2} y^{-2 H} / 2-y} \mathrm{~d} y, \quad x \neq 0 .
$$

The cumulative distribution function of $X(t)$ is

$$
F_{t}(x)=\int_{-\infty}^{x} f_{t}(y) \mathrm{d} y=\int_{0}^{\infty} \Phi\left(x y^{-H}\right) \frac{1}{\Gamma(t / v)} y^{t / v-1} \mathrm{e}^{-y} \mathrm{~d} y,
$$

where $\Phi(\cdot)$ is the cumulative distribution function of the standard normal distribution.

Remark 2.4. If $H=\frac{1}{2}$ then the above probability density function can be written as

$$
f_{t}(x)=\frac{\sqrt{2}}{\sqrt{\pi} \Gamma(t / v)}\left(\frac{|x|}{\sqrt{2}}\right)^{t / \nu-1 / 2} K_{t / v-1 / 2}(\sqrt{2}|x|), \quad x \neq 0,
$$

where $K_{\lambda}(\cdot)$ is the Bessel function of the third kind of index $\lambda$ [20, p. 190]. A closed form of the density is available when $t / v=n$ is an integer (see [31]), with $n=1$ producing the standard Laplace distribution.

The asymptotics of $f_{t}$ (mentioned in [21]) and $F_{t}$ are given in the following result.

Proposition 2.3. The density function of $X(t)$ admits the following asymptotic behavior:

$$
f_{t}(x) \sim a x^{(2 t / v) /(1+2 H)-1} \exp \left(-b x^{2 /(1+2 H)}\right), \quad x \rightarrow \infty,
$$

where

$$
a=\frac{1}{\sqrt{1+2 H} \Gamma(t / v)} H^{(t / v) /(1+2 H)-1 / 2} \text { and } b=\frac{1+2 H}{2} H^{-2 H /(1+2 H)} .
$$

The tail probability of $X(t)$ admits the following asymptotic behavior:

$$
\mathrm{P}(X(t)>x) \sim c x^{2(t / v-1) /(1+2 H)} \exp \left(-b x^{2 /(1+2 H)}\right), \quad x \rightarrow \infty,
$$

with $c=a H^{2 H /(1+2 H)}$. 


\subsection{The covariance structure}

The covariance function of $\{X(t), t \geq 0\} \stackrel{\mathrm{D}}{=} \mathcal{F} \mathcal{L} \mathcal{M}_{H}(\sigma, v)$ is easily derived from that of a FBM via a conditioning argument taking into account $\left|\Gamma_{t}-\Gamma_{s}\right| \stackrel{D}{=} \Gamma_{|t-s|}$ and well-known expressions for the moments of gamma distribution, leading to

$$
\mathrm{E}(X(t) X(s))=\frac{\sigma^{2}}{2}\left(\frac{\Gamma(2 H+t / v)}{\Gamma(t / v)}+\frac{\Gamma(2 H+s / v)}{\Gamma(s / v)}-\frac{\Gamma(2 H+|s-t| / v)}{\Gamma(|s-t| / v)}\right), \quad t \neq s .
$$

Our next result shows that when $t, s$, and $|s-t|$ are large, we essentially obtain the covariance structure of the FBM.

Proposition 2.4. If $G(s, t)$ and $F(s, t)$ are respectively the covariance functions of the $F B M$ given by (1.1) and the FLM given by (2.6), then, with $v=1, F(s, t) \sim G(s, t)$ as $|t| \rightarrow \infty$, $|s| \rightarrow \infty$, and $|s-t| \rightarrow \infty$.

\section{Fractional Laplace noise}

If $\{X(t), t \geq 0\} \stackrel{\mathrm{D}}{=} \mathcal{F} \mathcal{L} \mathcal{M}_{H}(\sigma, v)$ then for any $\eta>0$,

$$
\{Y(t), t \geq 0\} \stackrel{\mathrm{D}}{=}\{X(t+\eta)-X(t), t \geq 0\}
$$

is a stationary process with stationary increments. The process

$$
\left\{W_{j}, j \in \mathbb{N}\right\} \stackrel{\mathrm{D}}{=}\{Y(\eta(j-1)), j \in \mathbb{N}\}
$$

is called a fractional Laplace noise (FLN) with parameters $\sigma, v, \eta>0$, denoted by $\mathcal{F} \mathcal{L} \mathcal{N}_{H}(\sigma, v, \eta)$. The covariance function of the FLN, presented below, shows that, similarly to the fractional Brownian noise, an FLN exhibits a long-range dependence when $\frac{1}{2}<H<1$ [36].

We start with the covariance function of $\{Y(t), t \geq 0\}$. Clearly, for $s=t$ we have

$$
\mathrm{E}(Y(t) Y(s))=\operatorname{var}(Y(t))=\mathrm{E}\left([X(\eta)]^{2}\right)=\sigma^{2} \frac{\Gamma(2 H+\eta / v)}{\Gamma(\eta / v)} .
$$

The following two results provide the covariance function for $s \neq t$ and its asymptotic behavior.

Proposition 3.1. Let $\{Y(t), t \geq 0\}$ be the process defined by (3.1), where $X$ is an $\mathcal{F} \mathcal{L M}_{H}(\sigma, v)$. Then for any $t, s \geq 0$ with $s \neq t$, we have

$$
\begin{gathered}
\mathrm{E}(Y(t) Y(s))=\frac{\sigma^{2}}{2}\left(\frac{\Gamma(2 H+|s-t+\eta| / \nu)}{\Gamma(|s-t+\eta| / \nu)}+\frac{\Gamma(2 H+|t-s+\eta| / v)}{\Gamma(|t-s+\eta| / \nu)}\right. \\
\left.-2 \frac{\Gamma(2 H+|s-t| / v)}{\Gamma(|s-t| / \nu)}\right)
\end{gathered}
$$

if $\eta \neq|t-s|$, and

$$
\mathrm{E}(Y(t) Y(s))=\frac{\sigma^{2}}{2}\left(\frac{\Gamma(2 H+2 \eta / v)}{\Gamma(2 \eta / v)}-2 \frac{\Gamma(2 H+\eta / v)}{\Gamma(\eta / \nu)}\right)
$$

when $\eta=|s-t|$. 
Proposition 3.2. For each $t>0$, we have

$$
\mathrm{E}(Y(t) Y(s)) \sim \sigma^{2} H(2 H-1)\left(\frac{\eta}{v}\right)^{2}\left(\frac{s-t}{v}\right)^{2 H-2} \quad \text { as } s \rightarrow \infty .
$$

It follows directly from the above that the covariance function $r(n)=\mathrm{E}\left(W_{j} W_{j+n}\right)$ of the FLN $\left\{W_{j}, j \in \mathbb{N}\right\}$ is given by

$$
\begin{aligned}
& r(0)=\mathrm{E}\left([X(\eta)]^{2}\right)=\sigma^{2} \frac{\Gamma(2 H+\eta / \nu)}{\Gamma(\eta / v)}, \\
& r(n)=\frac{\sigma^{2}}{2}\left(\frac{\Gamma(2 H+(n+1) \eta / v)}{\Gamma((n+1) \eta / v)}+\frac{\Gamma(2 H+(n-1) \eta / v)}{\Gamma((n-1) \eta / v)}-2 \frac{\Gamma(2 H+n \eta / v)}{\Gamma(n \eta / v)}\right), \quad n \geq 1 .
\end{aligned}
$$

Moreover, for $H \neq \frac{1}{2}$ we have

$$
r(n) \sim \sigma^{2} H(2 H-1)\left(\frac{\eta}{v}\right)^{2 H} n^{2 H-2} \text { as } n \rightarrow \infty .
$$

Remark 3.1. Note that when $H>\frac{1}{2}$ and $n \rightarrow \infty$, the covariance function tends to 0 so slowly that the series $\sum_{n=1}^{\infty} r(n)$ diverges.

Remark 3.2. For $\eta=v$, the autocorrelation function is

$$
\rho(n)=\frac{H(2 H-1)}{\Gamma(2 H+1)} \frac{\Gamma(2 H+n-1)}{\Gamma(n+1)}, \quad n \geq 2,
$$

showing that the correlations are positive when $H>\frac{1}{2}$ and negative when $H<\frac{1}{2}$ (as in the case of FBM).

\section{Further properties}

\subsection{Infinite divisibility}

It is well known that variance mixtures of normal distributions are infinitely divisible whenever the distribution of the stochastic variance is infinitely divisible (see Property (e) of [12, Chapter XVII, Section 4]). When $H=\frac{1}{2}$, the stochastic variance $G_{t}^{2 H}$ has a gamma distribution, which is infinitely divisible. In the general case, we need to consider infinite divisibility of the generalized gamma distribution with probability density function (2.1), which corresponds to the $(1 / \gamma)$ th power of a $g(\alpha, 1)$-distributed variable. It is known that such powers are infinitely divisible whenever $0<|\gamma| \leq 1$, and are not infinitely divisible when $\gamma>1$ (the case $\gamma<-1$ is still open) [8], [38]. It follows that all marginal distributions of an FLM are infinitely divisible when $\frac{1}{2} \leq H \leq 1$. Since variance mixtures of normal distributions can be infinitely divisible even when the stochastic variance is not [19], the divisibility property for $H<\frac{1}{2}$ does not follow trivially. Our next result shows that we actually do not have infinite divisibility in this case.

Proposition 4.1. The marginal distributions of an $\mathcal{F} \mathcal{L} \mathcal{M}_{H}(\sigma, v)$ are infinitely divisible if and only if $\frac{1}{2} \leq H \leq 1$.

Remark 4.1. In the terminology of [9] and [37], when $\frac{1}{2} \leq H \leq 1$ the marginal distributions of an FLM are symmetric extended generalized gamma convolutions, i.e. variance mixtures of normal distributions where the stochastic variance is a generalized gamma convolution. These distributions are also self-decomposable [9, p. 107], [37]. 


\subsection{Connection with the abstract Cauchy problem}

A standard FLM $\{X(t)\}$ with $H=\frac{1}{2}$ is a Lévy process that induces the semigroup of operators $\left\{T_{t}, t \geq 0\right\}$, where

$$
T_{t} f(x)=\int f(x-s) f_{t}(s) \mathrm{d} s
$$

and $f_{t}$ is the (Bessel function) density of $X(t)$ given by (2.4) with $v=1$. The function

$$
q(x, t)=\int p(x-y) f_{t}(y) \mathrm{d} y
$$

solves an abstract Cauchy problem

$$
\frac{\partial q(x, t)}{\partial t}=L q(x, t)
$$

with the initial condition $q(x, 0)=p(x)$, where $L$ is the generator (of the semigroup) given by

$$
L f(x)=\int_{y \neq 0}\left(f(x-y)-f(x)-\frac{f^{\prime}(x) y}{1+y^{2}}\right) \phi(\mathrm{d} y)
$$

(see [2]). Here $\phi(\mathrm{d} y)$ is the Lévy measure of $X(1)$, which in our case is of the form $\phi(\mathrm{d} y)=$ $\exp (-\sqrt{2}|y|) /|y|[20$, p. 47]. Taking this into account, we find that the function $q(x, t)$ above solves the equation

$$
\frac{\partial q(x, t)}{\partial t}=\frac{1}{\lambda} E_{\lambda}^{+} q(x, t)+\frac{1}{\lambda} E_{\lambda}^{-} q(x, t),
$$

where $\lambda=\sqrt{2}$ and, for each $\lambda>0$, the quantities $E_{\lambda}^{+}$and $E_{\lambda}^{-}$are respectively right and left exponentially weighted derivative operators, defined as

$$
E_{\lambda}^{ \pm} f(x)=\int_{0}^{\infty} \frac{f(x-y) \mp f(x)}{y} \lambda \mathrm{e}^{-\lambda y} \mathrm{~d} y .
$$

If $\{X(t)\}$ is a Brownian motion, then $L=\partial^{2} / \partial x^{2}$ and (4.1) is the governing equation for classical diffusion. When $\{X(t)\}$ is an $\alpha$-stable Lévy motion, the generator $L$ involves fractional derivatives of order $\alpha$ with respect to the space variable $x$, and (4.1) becomes the fractional diffusion equation used in hydrology and physics to model anomalous diffusion (where the particles spread faster than in classical diffusion) [3], [4], [5], [28]. Fractional derivatives are defined similarly to the operators in (4.2), with the exponential weights replaced by a power function [34, pp. 109-110]. In fractional diffusion, the power function weights represent a particle flux that exceeds a given threshold $r$ with a probability that falls off like $r^{-\alpha}$. In situations in which the dynamics is governed by exponential probability tails (there is some evidence of this in chaotic dynamics [11]), a Laplace model may prove similarly useful.

\subsection{Stochastic self-similarity}

As mentioned in the introduction, FLM admits an interesting property, self-similarity, under random transformations of scale. In this section we formalize this notion and provide examples of stochastic processes that enjoy this property. Let $\left\{T_{c}(t), t \geq 0, c \in(1, \infty)\right\}$ be a family of stochastic processes, with $T_{c}(0)=0$ almost surely and nondecreasing sample paths, such that $\mathrm{E}\left(T_{c}(t)\right)=c t$ for each $t \geq 0$ and $c \in(1, \infty)$. This family corresponds to a stochastic time change, which on average is linear. In analogy to classical self-similarity, we define stochastic self-similarity as follows. 
Definition 4.1. Let $X=\{X(t), t \geq 0\}$ be a stochastic process on $\mathbb{R}^{d}$ and let

$$
T=\left\{T_{c}(t), t \geq 0, c \in(1, \infty)\right\}
$$

be a family of processes as described above, independent of $X$. The process $X$ is stochastically self-similar with index $H>0$ (or 'H-sss') with respect to the family $T$ if

$$
\left\{X\left(T_{c}(t)\right), t \geq 0\right\} \stackrel{\mathrm{D}}{=}\left\{c^{H} X(t), t \geq 0\right\}, \quad c \in(1, \infty) .
$$

For each $p \in(0,1)$, let $\left\{N_{p}(t), t \geq 0\right\}$ be the Lévy process such that $N_{p}(1)$ has the geometric distribution (1.2). This is a negative binomial process with a drift

$$
\left\{N_{p}(t), t \geq 0\right\} \stackrel{\mathrm{D}}{=}\left\{t+N B_{p}(t), t \geq 0\right\},
$$

where $N B_{p}$ is an integer-valued Lévy process with negative binomial marginal distributions given by

$$
\mathrm{P}\left(N B_{p}(t)=k\right)=\left(\begin{array}{c}
t+k-1 \\
k
\end{array}\right) p^{t}(1-p)^{k}, \quad k=0,1,2, \ldots, t>0 .
$$

Proposition 4.2. Let $\Gamma=\left\{\Gamma_{t}, t \geq 0\right\}$ be a gamma process with parameter $v$ and let $N_{p}$ be a negative binomial process with drift independent of $\Gamma$. Then $\Gamma$ is stochastically self-similar with index $H=1$ with respect to the family $T=\left\{T_{c}, c \in(1, \infty)\right\}$, where $T_{c}=\left\{v N_{1 / c}(t / v), t \geq 0\right\}$.

Corollary 4.1. Let $X=\{X(t), t \geq 0\}$ be an $\mathcal{F} \mathcal{L} \mathcal{M}_{H}(\sigma, v)$ and let $T$ be as in Proposition 4.2, independent of $X$. Then $X$ is $H$-sss with respect to the family $T$.

Remark 4.2. Corollary 4.1 can be generalized. If $Y=\{Y(t), t \geq 0\}$ is an $H$-ss process (i.e. self-similar with index $H$ ) and $\Gamma=\left\{\Gamma_{t}, t \geq 0\right\}$ is a gamma process independent of $Y$, then the compound process $X$ defined as $X(t)=Y\left(\Gamma_{t}\right)$ is $H$-sss with respect to the family $T$ above. This leads to a class of processes that are stochastically self-similar with respect to negative binomial changes of scale. For example, by taking $Y$ to be a stable $H$-ss process we obtain a corresponding geometric stable process with the stochastic self-similarity property. It is an interesting open question to find nontrivial examples of $H$-sss processes with respect to a different family of subordinators.

Remark 4.3. A completely different notion of stochastic self-similarity was proposed by Gupta and Waymire [14] and Veneziano [39]. Their notion involves stochastic renormalization in space, while ours involves stochastic renormalization in time. The two kinds of stochastic self-similarity are apparently unrelated.

\subsection{Stability properties of FLN}

Stochastic self-similarity properties of FLM lead to corresponding stability properties of FLN. For simplicity, we consider a standard FLM $X=\{X(t), t \geq 0\}$ with $v=\sigma=1$. With $t=1 / n$ in (4.3), in view of Corollary 4.1 we find that $(1 / p)^{H} X(1 / n) \stackrel{\mathrm{D}}{=} X\left(N_{p}(1 / n)\right)$, where $\left\{N_{p}(t), t \geq 0\right\}$ is the negative binomial process with drift described in Subsection 4.3. By writing this in terms of lag- $\eta$ increments of $X$ (see (3.2)), for $\eta=1 / n$ we obtain the relation

$$
p^{H} \sum_{j=1}^{n N_{p}(1 / n)} W_{j} \stackrel{\mathrm{D}}{=} W_{1},
$$


since $X\left(N_{p}(1 / n)\right)$ is the sum of the first $n N_{p}(1 / n)$ lag- $\eta$ increments $W_{j}$. If the lag size is one $(n=1)$, the number of terms in this sum is a geometric variable (1.2) and we obtain a generalization of the stability property (1.3) of the Laplace distribution.

Motivated by the last relation, we define a geometric renormalization group of transformations, $\left\{T_{p}, \circ\right\}$, and the corresponding concept of geometric self-similarity. Consider a stationary sequence $W=\left\{W_{j}, j \in \mathbb{N}\right\}$ and for each $p \in(0,1)$ define the transformation

$$
T_{p}: W \rightarrow T_{p} W=\left\{\left(T_{p} W\right)_{k}, k \in \mathbb{N}\right\},
$$

where

$$
\left(T_{p} W\right)_{k}=p^{H}\left(W_{N_{p}(k-1)+1}+\cdots+W_{N_{p}(k)}\right), \quad k \in \mathbb{N} .
$$

As before, $N_{p}(t)$ is a negative binomial process with drift, independent of $W$. In words, $T_{p}$ transforms the original sequence into a new one obtained by summing the components of $W$ over successive blocks of random geometric size $N_{p}^{(k)}=N_{p}(k)-N_{p}(k-1)$ and then normalizing with $p^{H}=(\text { expected block size })^{H}$. The group operation is composition, satisfying

$$
T_{p} \circ T_{q} W=T_{p}\left(T_{q} W\right) \stackrel{\mathrm{D}}{=} T_{p q} W
$$

since, for two independent negative binomial processes with respective drifts $N_{p}$ and $N_{q}$, we have $N_{p q}(k)=N_{p}\left(N_{q}(k)\right), k \in \mathbb{N}$. Now, for the lag-1 stationary FLN, $W=\left\{W_{j}, j \in \mathbb{N}\right\}$, we notice that $W_{N_{p}(k-1)+1}+\cdots+W_{N_{p}(k)}=X\left(N_{p}(k)\right)-X\left(N_{p}(k-1)\right)$. Thus, stochastic self-similarity of $X$, discussed in Corollary 4.1, implies that $W$ is a fixed point of the geometric renormalization group $\left\{T_{p}\right.$, o $\}$, i.e. that $T_{p} W \stackrel{\mathrm{D}}{=} W, p \in(0,1)$. This motivates the following definition.

Definition 4.2. If $W=\left\{W_{j}, j \in \mathbb{N}\right\}$ is a stationary sequence and $T_{p} W \stackrel{\mathrm{D}}{=} W$, then we say that $W$ is geometrically self-similar with index $H$.

Proposition 4.3. The lag-1 FLN corresponding to an FLM with $v=1$ is geometrically selfsimilar with index $H$.

Remark 4.4. The above notion of geometric self-similarity as applied to stationary sequences is similar to invariance properties of renewal processes with respect to geometric thinning [13], [32], since both properties are based on stability with respect to geometric compounding.

\section{Simulation}

The most direct approach to simulating sample paths of FLM is by using a subordination of a fractional Brownian motion to a gamma process. For $H>\frac{3}{4}$ and large-to-moderate values of $v$, we find it adequate to simulate a fractional Brownian motion on a regular grid and subordinate it to a discretized gamma process. For other parameter values we generate values of $\Gamma\left(t_{k}\right)$ for points $t_{k}$ on an equally spaced grid. Then we simulate $X_{k}=B_{H}\left(\Gamma\left(t_{k}\right)\right)$, which, conditionally on the values of $\Gamma\left(t_{k}\right)$, is a second-order Gaussian process with the explicit covariance function of FBM evaluated at the values of the gamma process (note that in this case $X_{k}$ is not a stationary sequence). To generate the second-order Gaussian sequence $X_{k}$ we use the innovations algorithm to represent the sequence as $X_{k}=\alpha_{1 k} Z_{1}+\cdots+\alpha_{k k} Z_{k}$, where the $Z_{i}$ are independent, identically distributed standard normal variables and the $\alpha_{i j}$ are computed using the innovations algorithm [10, p. 172]. Figure 1 shows some representative sample paths of FLMs. The graphs show how the time scale parameter $v$ affects the degree of jump-type behavior for different degrees of similarity to FBM. In the upper graph $H=0.3$ and in the 

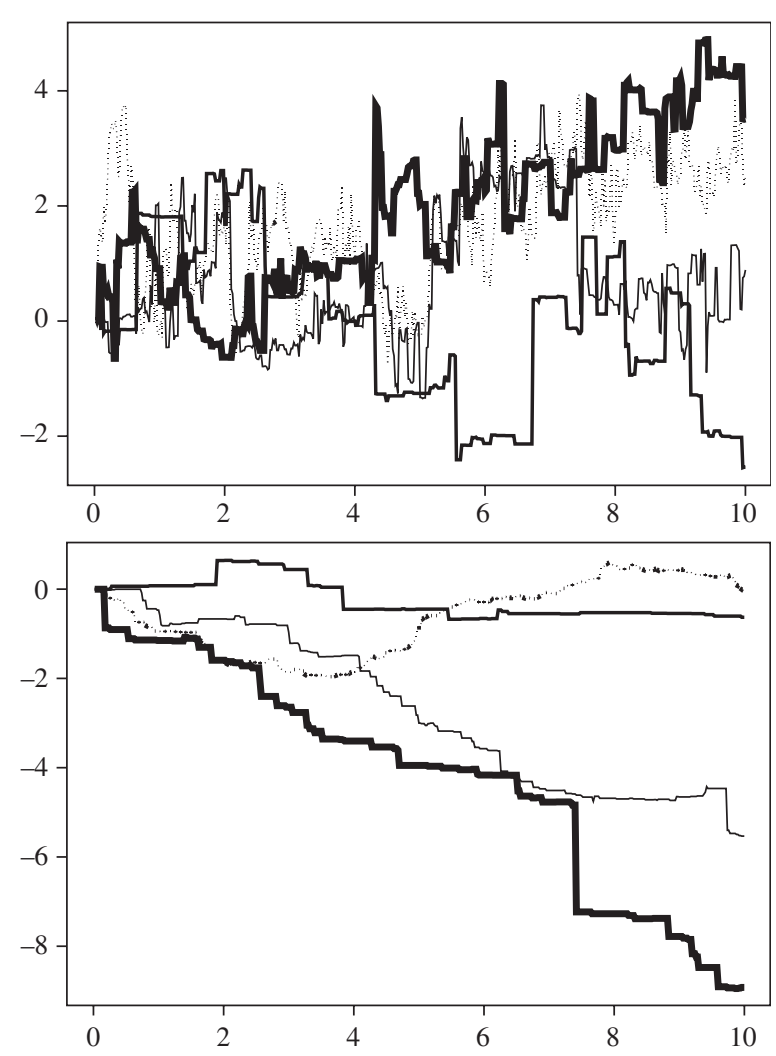

FIGURE 1: Trajectories of FLM for different values of the self-similarity parameter, $H$, and time scale parameter, $v$.

lower graph $H=0.8$, while in each graph $v=1$ for the solid line of medium weight, $v=\frac{1}{2}$ for the heavy solid line, $v=\frac{1}{4}$ for the light solid line, and $v=\frac{1}{16}$ for the dotted line.

\section{Proofs}

Proof of Proposition 2.3. Making the substitution $\tau=y^{-2 H} / 2$ in (2.3) allows us to write

$$
f_{t}(x)=\frac{1}{H} 2^{-t /(2 H v)-1 / 2} h\left(x^{2}\right), \quad x \neq 0
$$

where

$$
h(\rho)=\int_{0}^{\infty} \tau^{\gamma-1} \mathrm{e}^{-\rho \tau-\alpha \tau^{-\beta}} \mathrm{d} \tau
$$

with $\alpha=2^{-1 /(2 H)}, \beta=1 /(2 H)$, and $\gamma=[1-t /(H v)] / 2$. The statement of the proposition now follows from the asymptotic relation

$$
h(\rho) \sim\left(\frac{\alpha \beta}{\rho}\right)^{\gamma /(\beta+1)} \mathrm{e}^{-\lambda(1+1 / \beta)} \sqrt{\frac{2 \pi}{\beta+1}} \lambda^{-1 / 2}, \quad \rho \rightarrow \infty,
$$

where $\lambda=\left(\alpha \beta \rho^{\beta}\right)^{1 /(\beta+1)}$ (see [40, p. 62, Example 2]). 
The tail probability asymptotics is obtained via l'Hôpital's rule applied to the ratio of the sides of (2.5).

Proof of Proposition 2.4. It suffices to consider the case in which $0<s<t$ and $s \rightarrow \infty$. By Stirling's formula, we have $x^{-2 H} \Gamma(x+2 H) / \Gamma(x) \rightarrow 1$ as $x \rightarrow \infty$. We now consider three cases.

Case 1. Suppose that $t \sim \lambda s$ for some $\lambda>1$. Then $(t-s) \sim(\lambda-1) s \rightarrow \infty$, meaning that $G(s, t) \sim\left[s^{2 H}\left(\lambda^{2 H}+1-(\lambda-1)^{2 H}\right)\right] / 2$ and, thus,

$$
\begin{aligned}
\frac{F(s, t)}{G(s, t)} \sim \frac{1}{\lambda^{2 H}+1-(\lambda-1)^{2 H}} & \left(\lambda^{2 H} t^{-2 H} \frac{\Gamma(t+2 H)}{\Gamma(t)}+s^{-2 H} \frac{\Gamma(s+2 H)}{\Gamma(s)}\right. \\
& \left.-(\lambda-1)^{2 H}(t-s)^{-2 H} \frac{\Gamma(t-s+2 H)}{\Gamma(t-s)}\right) \rightarrow 1 .
\end{aligned}
$$

Case 2. Suppose that $(t-s) / t \rightarrow 0$. Then $t \sim s$, meaning that $G(s, t) \sim t^{2 H}$ and, thus,

$$
\frac{F(s, t)}{G(s, t)} \sim \frac{1}{2}\left(\frac{\Gamma(t+2 H)}{t^{2 H} \Gamma(t)}+\frac{\Gamma(s+2 H)}{s^{2 H} \Gamma(s)}-\left(\frac{t-s}{t}\right)^{2 H} \frac{\Gamma(t-s+2 H)}{(t-s)^{2 H} \Gamma(t-s)}\right) \rightarrow 1 .
$$

Case 3. Suppose that $s / t \rightarrow 0$. Then $(t-s) / t \rightarrow 1$, meaning that

$$
G(s, t) \sim \frac{1}{2} t^{2 H}\left[1-\left(1-\frac{s}{t}\right)^{2 H}\right]=\frac{1}{2} t^{2 H}\left[1-\left\{1-2 H\left(\frac{s}{t}\right)+o\left(\frac{s}{t}\right)\right\}\right] \sim H s t^{2 H-1}
$$

and, thus, with $\Psi(t)=\mathrm{d} \ln (\Gamma(t)) / \mathrm{d} t$ the digamma function,

$$
\begin{aligned}
\frac{F(s, t)}{G(s, t)} & \sim \frac{t}{H s} t^{-2 H} \frac{\Gamma(t+2 H)}{\Gamma(t)} \frac{1}{2}\left(1-\frac{\Gamma(t)}{\Gamma(t+2 H)} \frac{\Gamma(t-s+2 H)}{\Gamma(t-s)}\right) \\
& =\frac{t}{H s} t^{-2 H} \frac{\Gamma(t+2 H)}{\Gamma(t)} \frac{1}{2}\left(1-\left\{1-(\Psi(t+2 H)-\Psi(t)) s+o\left(\frac{s}{t}\right)\right\}\right) \\
& \sim \frac{t}{H s} \frac{H s}{t}=1,
\end{aligned}
$$

using a Taylor expansion and the fact that $\Psi(t+2 H)-\Psi(t) \sim 2 H / t$.

In the general case, given any two sequences $s_{n} \rightarrow \infty$ and $t_{n}>s_{n}$, any subsequence $\left(n^{\prime}\right)$ contains a further subsequence, $\left(n^{\prime \prime}\right)$, along which $s_{n^{\prime \prime}} / t_{n^{\prime \prime}} \rightarrow \mu$ for some $\mu \in[0,1]$. One of the three cases above then implies that $F\left(s_{n^{\prime \prime}}, t_{n^{\prime \prime}}\right) / G\left(s_{n^{\prime \prime}}, t_{n^{\prime \prime}}\right) \rightarrow 1$ and, since this is true for every subsequence, it follows that $F\left(s_{n}, t_{n}\right) / G\left(s_{n}, t_{n}\right) \rightarrow 1$ as $n \rightarrow \infty$, which proves the claim.

Proof of Proposition 3.1. Write

$\mathrm{E}(Y(t) Y(s))=\mathrm{E}(X(t+\eta) X(s+\eta))+\mathrm{E}(X(t) X(s))-\mathrm{E}(X(t+\eta) X(s))-\mathrm{E}(X(t) X(s+\eta))$

and use the formulae for the covariance function of $\{X(t), t \geq 0\}$ presented in Section 2.

Proof of Proposition 3.2. For $r>h>0$, let

$$
F(r, h)=\frac{1}{2}\left[\frac{\Gamma(r+h+2 H)}{\Gamma(r+h)}+\frac{\Gamma(r-h+2 H)}{\Gamma(r-h)}-2 \frac{\Gamma(r+2 H)}{\Gamma(r)}\right] .
$$


It is enough to show that for each $h>0$ we have $F(r, h) \sim H(2 H-1) h^{2} r^{2 H-2}$ as $r \rightarrow \infty$ (then, to obtain the result desired here, set $r=(s-t) / v$ and $h=\eta / v)$.

Let $g_{t}=\Gamma(t+2 H) / \Gamma(t)$. Using Taylor expansions yields

$$
\begin{aligned}
\frac{g_{r+h}}{g_{r}}= & 1+(\Psi(r+2 H)-\Psi(r)) h+\frac{(\Psi(r+2 H)-\Psi(r))^{2}+\Psi_{1}(r+2 H)-\Psi_{1}(r)}{2} h^{2} \\
& +O\left(r^{-3}\right)
\end{aligned}
$$

where $\Psi(x)=\mathrm{d} \ln (\Gamma(x)) / \mathrm{d} x$ and $\Psi_{1}(x)=\mathrm{d} \Psi(x) / \mathrm{d} x$ are the digamma and trigamma functions, respectively. We have

$$
\begin{gathered}
\Psi(x)=\ln x+\frac{1}{2 x}+\sum_{n=1}^{\infty} \frac{B_{2 n}}{2 n x^{2 n}}, \\
\Psi_{1}(x)=\frac{1}{x}-\frac{1}{2 x^{2}}-\sum_{n=1}^{\infty} \frac{B_{2 n}}{x^{2 n+1}},
\end{gathered}
$$

where the $B_{2 n}$ s are the Bernoulli numbers. Thus, $\Psi(r+2 H)-\Psi(r) \sim 2 H / r$ and

$$
\Psi_{1}(r+2 H)-\Psi_{1}(r)=\frac{-2 H}{r(r+2 H)}+O\left(r^{-3}\right),
$$

whence

$$
\frac{g_{r+h}}{g_{r}}=1+2 H \frac{h}{r}+H(2 H-1)\left(\frac{h}{r}\right)^{2}+O\left(r^{-3}\right) .
$$

Finally,

$$
\begin{aligned}
F(r, h) & =\frac{g_{r}}{2}\left[\frac{g_{r+h}}{g_{r}}+\frac{g_{r-h}}{g_{r}}-2\right] \\
& =g_{r}\left[\frac{H(2 H-1)}{r^{2}} h^{2}+O\left(r^{-3}\right)\right] \\
& \sim H(2 H-1) h^{2} r^{2 H-2} .
\end{aligned}
$$

Proof of Proposition 4.1. In view of the remarks preceding Proposition 4.1, it is enough to show that $X(t)$ is not infinitely divisible when $H<\frac{1}{2}$. To see this, assume the contrary and use Proposition 2.3 to conclude that the limit

$$
\lim _{x \rightarrow \infty}-\frac{\log \mathrm{P}(X(t)>x)}{x \log x}
$$

is the same as $\lim _{x \rightarrow \infty} b x^{\beta} /(x \log x)$, where

$$
\beta=\frac{2}{1+2 H} \quad \text { and } \quad b=\frac{1}{2}(1+2 H) H^{-2 H /(1+2 H)} .
$$

Since $\beta>1$ when $H<\frac{1}{2}$, the limit is infinite. Since $X(t)$ is not degenerate, this would imply that $X(t)$ is Gaussian (see [35, p. 200, Corollary 9.9]). This contradiction shows that $X(t)$ cannot be infinitely divisible in this case. 
Proof of Proposition 4.2. Since $\Gamma\left(v N_{1 / c}(t / v)\right)$ and $\Gamma(t) / p$ are Lévy processes, it is enough to show that for some $t>0$ the random variables $\Gamma\left(v N_{1 / c}(t / v)\right)$ and $\Gamma(t) / p$ have the same distribution. For $t=v$ we find that $\Gamma(t)$ is a standard exponential random variable and that $\Gamma\left(v N_{1 / c}(t / v)\right)$ is the sum of a geometric number, $N_{1 / c}(1)$, of independent standard exponential random variables. The latter has the characteristic function

$$
\phi(t)=\sum_{k=1}^{\infty} \frac{1}{(1-\mathrm{i} t)^{k}}(1-p)^{k-1} p=\frac{p}{(1-\mathrm{i} t)-(1-p)}=\frac{1}{1-\mathrm{i} t / p},
$$

which corresponds to an exponential random variable multiplied by $1 / p$.

\section{Acknowledgements}

We thank the two referees and a member of the Editorial Board for their valuable comments and additional references.

\section{References}

[1] Amoroso, L. (1925). Ricerche intorno alla curva dei redditi. Ann. Mat. Pura Appl. Ser. 4 21, 123-159.

[2] Baeumer, B. And Meerschaert, M. M. (2001). Stochastic solutions for fractional Cauchy problems. Fract. Calc. Appl. Anal. 4, 481-500.

[3] Benson, D. A., Wheatcraft, S. W. and Meerschaert, M. M. (2000). Application of a fractional advectiondispersion equation. Water Resources Res. 36, 1403-1412.

[4] Benson, D. A., Wheatcraft, S. W. and Meerschaert, M. M. (2000). The fractional-order governing equation of Lévy motion. Water Resources Res. 36, 1413-1424.

[5] Benson, D. A., Schumer, R., Meerschaert, M. M. and Wheatcraft, S. W. (2001). Fractional dispersion, Lévy motions, and the MADE tracer tests. Transport Porous Media 42, 211-240.

[6] Beran, J. (1994). Statistics for Long-Memory Processes. Chapman and Hall, New York.

[7] Bibby, B. M. And Sørensen, M. (2003). Hyperbolic distributions in finance. In Handbook of Heavy Tailed Distributions in Finance, ed. S. T. Rachev, Elsevier, Amsterdam, pp. 211-248.

[8] Bondesson, L. (1979). A general result on infinite divisibility. Ann. Prob. 7, 965-979.

[9] Bondesson, L. (1992). Generalized Gamma Convolutions and Related Classes of Distributions and Densities (Lecture Notes Statist. 76). Springer, New York.

[10] Brockwell, P. J. And Davis, R. A. (1991). Time Series: Theory and Methods. Springer, New York.

[11] Ching, E. S. And Tu, Y. (1994). Passive scalar fluctuations with and without a mean gradient: a numerical study. Phys. Rev. E 49, 1278-1282.

[12] Feller, V. (1971). Introduction to the Theory of Probability and Its Applications, Vol. 2, 2nd edn. John Wiley, New York.

[13] GnedenKo, B. V. (1970). Limit theorems for sums of a random number of positive independent random variables. In Proc. 6th Berkeley Symp. Math. Statist. Probab., Vol. 2, University of California Press, Berkeley, pp. 537-549.

[14] Gupta, V. K. And Waymire, E. C. (1990). Multiscaling properties of spatial rainfall in river flow distributions. J. Geophys. Res. 95, 1999-2009.

[15] Heyde, C. C. (1999). A risky asset model with strong dependence through fractal activity time. J. Appl. Prob. 36, 1234-1239.

[16] Heyde, C. C. (2002). On modes of long-range dependence. J. Appl. Prob. 39, 882-888.

[17] Heyde, C. C. And Yang, Y. (1997). On defining long-range dependence. J. Appl. Prob. 34, 939-944.

[18] Johnson, N. L., Kotz, S. and Balakrishnan, B. (1994). Continuous Univariate Distributions, Vol. 1, 2nd edn. John Wiley, New York.

[19] KelKer, D. (1971). Infinite divisibility and variance mixtures of the normal distribution. Ann. Math. Statist. 42, 802-808.

[20] Kotz, S., Kozubowski, T. J. And Podgórski, K. (2001). The Laplace Distribution and Generalizations: A Revisit with Applications to Communications, Economics, Engineering, and Finance. Birkhäuser, Boston, MA.

[21] Levin, A. AND TCHERnitser, A. (2003). Multifactor stochastic variance models in risk management: maximum entropy approach and Lévy processes. In Handbook of Heavy Tailed Distributions in Finance, ed. S. T. Rachev, Elsevier, Amsterdam, pp. 443-480.

[22] Madan, D. B., CARR, P. P. And Chang, E. C. (1998). The variance gamma process and option pricing. Europ. Fin. Rev. 2, 74-105. 
[23] Madan, D. B. and Seneta, E. (1990). The variance-gamma (V.G.) model for share markets returns. J. Business 63, 511-524.

[24] Maejima, M. And Rosiński, J. (2001). The class of type $G$ distributions on $\mathbb{R}^{d}$ and related subclasses of infinitely divisible distributions. Demonstr. Math. 34, 251-266.

[25] Maejima, M. and Rosiński, J. (2002). Type G distributions on $\mathbb{R}^{d}$. J. Theoret. Prob. 15, 323-341.

[26] Marcus, M. B. (1987). $\xi$-radial Processes and Random Fourier Series (Mem. Ser. 68). American Mathematical Society, Providence, RI.

[27] Meerschaert, M. M., Kozubowski, T. J., Molz, F. J. And Lu, S. (2004). Fractional Laplace model for hydraulic conductivity. Geophys. Res. Lett. 31, L08501.

[28] Metzler, R. AND Klafter, J. (2000). The random walk's guide to anomalous diffusion: a fractional dynamics approach. Phys. Rep. 339, 1-77.

[29] Molz, F. J., Kozubowski, T. J., Podgórski, K. and Castle, J. W. (2005). A generalization of the fractal/facies model. Preprint.

[30] Molz, F. J., Meerschaert, M. M., Kozubowski, T. J., and Hyden, P. D. (2005). Do heterogeneous sediment properties and turbulent velocity fluctuations have something in common? Some history and a new stochastic process. In Dynamics of Fluids and Transport in Fractured Rock (Geophys. Monogr. Ser. 162), eds B. Faybishenko, P. A. Witherspoon and J. Gale, American Geophysical Union, Washington, DC, pp. 13-22.

[31] Press, S. J. (1967). On the sample covariance from a bivariate normal distribution. Ann. Inst. Statist. Math. 19, 355-361.

[32] RÉNYI, A. (1976). A characterization of Poisson processes. In Selected Papers of Alfréd Rényi, Vol. 1, ed. P. Turán, Akadémiai Kiadó, Budapest, pp. 622-628.

[33] RosińsKI, J. (1991). On a class of infinitely divisible processes represented as mixtures of Gaussian processes. In Stable Processes and Related Topics, eds S. Cambanis, G. Samorodnitsky and M. S. Taqqu, Birkhäuser, Boston, MA, pp. 27-41.

[34] Samko, S. G., Kilbas, A. A. and Marichev, O. I. (1993). Fractional Integrals and Derivatives: Theory and Applications. Gordon and Breach, London.

[35] Steutel, F. W. And van Harn, K. (2004). Infinite Divisibility of Probability Distributions on the Real Line. Marcel Dekker, New York.

[36] TAQQU, M. S. (2003). Fractional Brownian motion and long-range dependence. In Theory and Applications of Long-Range Dependence, eds P. Doukhan, G. Oppenheim and M. S. Taqqu, Birkhäuser, Boston, MA, pp. 5-38.

[37] Thorin, O. (1978). An extension of the notion of a generalized gamma convolution. Scand. Actuar. J. 1978, 141-149.

[38] Thorin, O. (1978). Proof of a conjecture of L. Bondesson concerning infinite divisibility of powers of a gamma variable. Scand. Actuar. J. 1978, 151-164.

[39] Veneziano, D. (1999). Basic properties and characterization of stochastically self-similar processes in $\mathbb{R}^{d}$. Fractals 7, 59-78.

[40] Wong, R. (1989). Asymptotic Approximations of Integrals. Academic Press, Boston, MA. 